\title{
Plasma levels of leptin and ghrelin and their correlation with BMI, and circulating lipids and glucose in obese Tunisian women
}

\author{
Fethi Ben Slamaa , Nahawand Jridi ${ }^{\mathrm{b}}$, Mohamed Chiheb Ben Rayanac ${ }^{\mathrm{c}}$, Abdelmagid Trimechec, \\ Mohamed Hsairi ${ }^{\mathrm{a}}$, Omrane Belhadj ${ }^{\mathrm{d}}$ \\ ${ }^{a}$ National Institute of Public Health (INSP), Ministry of Public Health, Tunis, Tunisia \\ ${ }^{b}$ Faculty of Pharmacy, University of Monastir, Tunisia \\ ${ }^{c}$ National Institute of Nutrition and Food Technology (INNTA), Ministry of Public Health, Tunis, \\ Tunisia \\ ${ }^{d}$ Biochemistry and Biotechnology Laboratory, Faculty of Sciences of Tunis, Tunis El Manar University, \\ Tunisia
}

\begin{abstract}
Background: A National Survey on the Effect of Nutrition conducted in 1996-1997 showed a 14\% overall prevalence of obesity in Tunisia with a female predominance. Leptin and ghrelin play crucial roles in regulating body weight and energy balance. Leptin, an anorectic hormone, and ghrelin, an orexigenic hormone, appear to interact with glucose and lipid metabolism.

Objectives: To determine the circulating levels of ghrelin and leptin in obese Tunisian women and to investigate the correlations of these hormones with body mass index (BMI), and circulating lipids and glucose.

Methods: Forty obese women were recruited from patients in the " $C$ " Unit of the National Institute of Nutrition and Food Technology. Twenty normal women were recruited as controls. Plasma levels of the studied variables were measured in patients from both groups and findings were analyzed.

Results: Circulating levels of leptin were significantly higher, while high-density lipoprotein (HDL)-cholesterol and ghrelin levels were significantly lower in the obese women. In the obese women, significant positive correlations were found between circulating levels of leptin and low-density lipoprotein (LDL)-cholesterol, BMI, and glucose; and ghrelin and HDL-cholesterol. Significant negative correlations were found between circulating levels of leptin and HDL-cholesterol and ghrelin; and ghrelin and leptin, LDL-cholesterol, BMI, and glucose. Multivariate analysis revealed that ghrelin was significantly associated with HDL-cholesterol, LDL-cholesterol, and blood glucose.

Conclusions: The significant negative correlation between leptin and ghrelin suggests that these two hormones may be antagonistic. Increased levels of ghrelin are correlated with decreased circulating levels of HDL-cholesterol and increased levels of LDL-cholesterol.
\end{abstract}

Keywords: BMI, ghrelin, leptin, lipids, obesity

Obesity is a major public health problem given the complications and socioeconomic impact it causes [1-3]. Approximately 500 million people worldwide suffer from obesity with a significantly female predominance. According to the latest World Health Organization estimates, obesity affects $16.4 \%$ of the population in Australia, 16.9\% in France, 2.9\% in China, 30.3\% in Egypt, 35.6\% in Saudi Arabia, and

Correspondence to: Fethi Ben Slama, National Institute of Public Health, Boulevard Hedi Saidi, Bab Saadoun, 1005 Tunis, Tunisia.E-mail: fethi.benslama@rns.tn
$0.5 \%$ in Vietnam $[4,5]$. Tunisia is undergoing epidemiological transition to chronic diseases and malnutrition, which are reflected in a rapid increase in the prevalence of obesity [6]. The National Survey effect of Nutrition conducted in 1996-1997 showed that the overall prevalence of obesity in Tunisia is in the order of $14 \%$ with a female predominance [6]. The discovery of leptin in 1994 and ghrelin in 1999 increased understanding the pathophysiology of obesity and provided new opportunities for the treatment and fight against this disease [7-9]. Indeed, these two hormones, with their opposing properties, play a crucial 
role in regulating body weight and energy balance. The adipokine leptin is synthesized by adipose tissue in proportion to fat mass and is an anorectic hormone contributing to the inhibition of appetite and increase of energy expenditure. Ghrelin, synthesized by the stomach, is the only known peripheral orexigenic hormone [9]. Leptin and ghrelin appear to interact with glucose and lipid metabolism [10-12]. Leptin has been well studied. For developing countries, we are among the first, in Tunisia, to study the levels of ghrelin in obese patients.

The objectives of this work were to determine through a case-control study the levels of leptin, ghrelin, and lipid variables in a group of obese women compared with a control group of healthy women; and to explore the existence of possible correlations between leptin, ghrelin, BMI, and lipid variables among Tunisians.

\section{Materials and methods Population}

After approval of the National Ethics Committee for the implementation of this study, we recruited 40 obese women and 20 female controls. They were matched by age. Obese women were recruited from nursing unit "C" of the National Institute of Nutrition and Food Technology (INNTA). A woman was considered obese when her body mass index (BMI) was greater than 30 . Women selected for the survey were informed in advance about study objectives and showed no pathology or metabolic complication other than obesity. The healthy control group was recruited from women accompanying patients and who also agreed to participate in the survey. As motivation, they received results of their conventional laboratory tests, all gave their written consent to participate. It should be noted that, at the beginning of our investigation, we had the agreement of 45 women who were considered healthy after physical examination by the team physician, but 25 women withdrew during the blood taking phase.

\section{Data collection}

We used a form to collect anthropometric data. Each woman was weighed 2 consecutive times using a calibrated scale and their height was measured. The average of these measurements was calculated. Blood samples were taken from an antecubital vein after a 12 hour fast. The measured biochemical variables were: total cholesterol (TC), triglycerides (TG), highdensity lipoprotein-cholesterol (HDL-C), fasting blood glucose, and low-density lipoprotein-cholesterol (LDL-C). Blood glucose determination was by an enzymatic glucose oxidase method using a Beckman kit on Beckman Synchron CX9 analyzer. TGs were determined using a Beckman Synchron Cx7 kit. (Beckman Coulter, Brea, CA, USA). TC was measured using a cholesterol oxidase kit adapted to a Beckman Synchron CX9 analyzer. The HDLcholesterol was determined using a Randox kit with a Beckman Synchron CX9. LDL-cholesterol was calculated using the Friedwald formula [LDL $=\mathrm{CT}-$ (TG/5 + HDL-C) with the condition TG $<4$ g/L]. Ghrelin was measured using a radioimmunoassay (GHRA-88HK, Linco Research, Inc, MO, USA) for active ghrelin with a sensitivity of $7.8 \mathrm{pg} / \mathrm{mL}$. Leptin was measured using a radioimmunoassay (HL-81HK, Millipore) with a sensitivity ranging from $0.5 \mathrm{ng} / \mathrm{mL}$ to $100 \mathrm{ng} / \mathrm{mL}$.

\section{Statistical analysis}

Data were analyzed with SPSS software, version 11.5 (SPSS Inc, Chicago, IL, USA). Comparisons of distributions of two numeric variables were conducted using a nonparametric Mann-Whitney $U$ test. Correlations were tested using a Pearson correlation coefficient and a Spearman rank-correlation coefficient.

To determine the threshold-value of ghrelin that increases the risk of obesity, we have established a receiver operating characteristics (ROC) curve. After verifying that the area under the curve was significant $(>0.50)$, we have identified the value of ghrelin, which maximizes the pair "sensitivity-specificity". We calculated the odds ratio, associated with this value.

Multivariate backward linear regression analysis was conducted with ghrelin as a dependent variable and BMI, HDL-cholesterol, LDL-cholesterol, glucose, and leptin as independent variables. For all tests, $P<0.05$ was considered significant.

\section{Results}

As shown in Table 1, we found that the level of leptin was significantly higher in obese women. Similarly, the levels of TC and TG were higher in obese women, but the difference was not statistically significant. The levels of HDL-cholesterol and ghrelin were significantly higher in women in the control group. 
Table 1. Comparison of mean lipid parameters, ghrelin, and leptin in obese women and controls

\begin{tabular}{lccc}
\hline Parameters & $\begin{array}{c}\text { Obese women } \\
\text { (Mean } \pm \text { SD) }\end{array}$ & $\begin{array}{c}\text { Controls } \\
\text { (Mean } \pm \text { SD) }\end{array}$ & $P$ \\
\hline Total cholesterol (mmol/L) & $5.04 \pm 1.10$ & $4.52 \pm 1.05$ & 0.08 \\
HDL-cholesterol (mmol/L) & $1.03 \pm 0.25$ & $1.19 \pm 0.14$ & $<0.01$ \\
LDL-cholesterol (mmol/L) & $3.37 \pm 0.61$ & $2.70 \pm 0.83$ & $<0.001$ \\
Triglycerides (mmol/L) & $1.95 \pm 0.84$ & $1.51 \pm 0.91$ & 0.06 \\
Glucose (mmol/L) & $7.95 \pm 0.52$ & $5.91 \pm 1.05$ & $<0.001$ \\
Leptin (ng/mL) & $19.35 \pm 5.78$ & $4.68 \pm 1.88$ & $<0.001$ \\
Ghrelin (pg/mL) & $29.63 \pm 9.62$ & $56.02 \pm 23.60$ & $<0.001$ \\
Total & 40 & 20 & \\
\hline
\end{tabular}

HDL = high-density lipoprotein, LDL = low-density lipoprotein, SD = standard deviation

Correlations between leptin, ghrelin, and other measured biochemical variables in obese women group are summarized in Table 2. Leptin levels in the obese women were positively correlated with LDLcholesterol, BMI, and blood glucose, and negatively correlated with HDL-cholesterol. Ghrelin levels were negatively correlated with blood glucose (Figure 1), BMI, and LDL-cholesterol, and positively correlated with HDL-cholesterol (Figure 2). Ghrelin levels were negatively correlated with leptin levels (Figure 3).

Table 2. Correlations between leptin, ghrelin, and other parameters

\begin{tabular}{llllllll}
\hline & Leptin & HDL-chol & LDL-chol & TG & Ghrelin & BMI & Glucose \\
\hline \multirow{2}{*}{ Leptin } & - & $r=-0.48$ & $r=0.48$ & $r=-0.051$ & $r=-0.5$ & $r=0.61$ & $r=0.42$ \\
Ghrelin & - & $P=0.002$ & $P=0.002$ & $P=0.58$ & $P=0.01$ & $P<0.001$ & $P=0.008$ \\
& $r=-0.5$ & $\mathrm{r}=0.64$ & $\mathrm{r}=-0.423$ & $r=-0.08$ & - & $r=-0.41$ & $r=-0.49$ \\
& $P=0.001$ & $P<0.001$ & $P=0.007$ & $P=0.61$ & - & $P=0.01$ & $P=0.001$
\end{tabular}

HDL-chol = high-density lipoprotein cholesterol, LDL-chol = low-density lipoprotein cholesterol, TG = triglycerides, $\mathrm{BMI}=$ body mass index

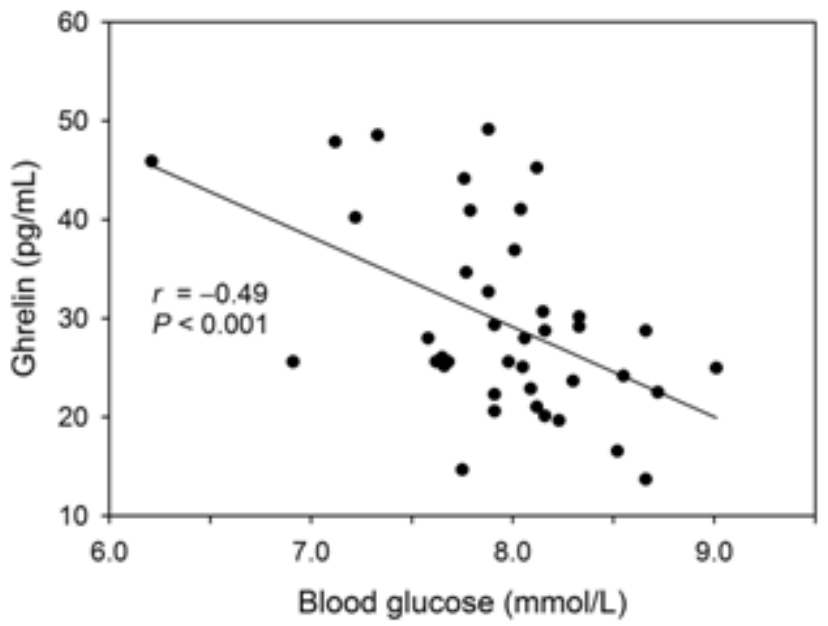

Figure 1. Correlation between ghrelin and blood glucose in obese Tunisian women 


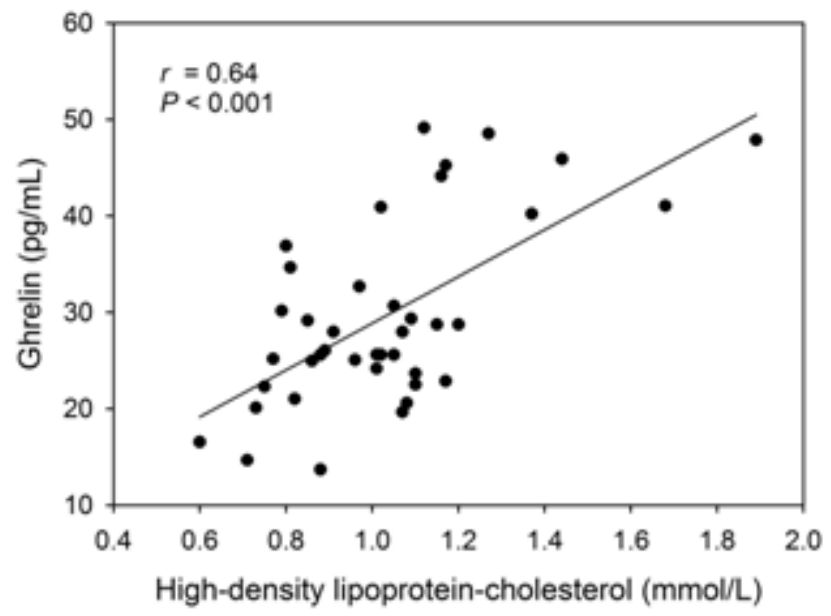

Figure 2. Correlation between ghrelin and high-density lipoprotein-cholesterol in obese Tunisian women

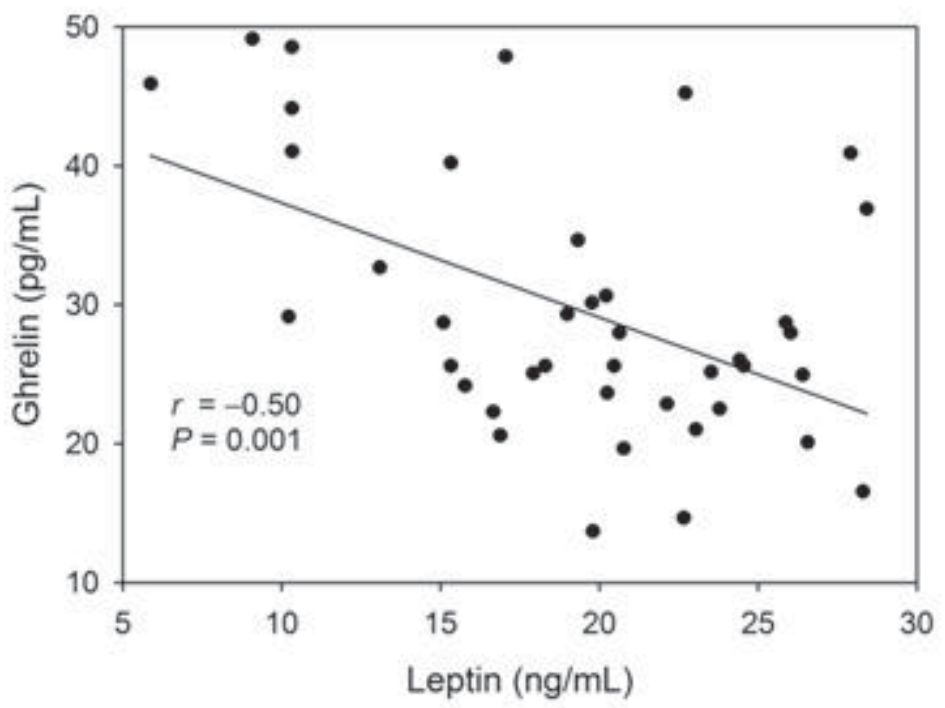

Figure 3. Correlation between ghrelin and leptin in obese Tunisian women

Following the univariate study, we used a ROC curve (Figure 4) to determine the threshold below which the ghrelin may increase and worsen body weight. Respecting the applicability criteria of the curve (the area below the curve is significantly $>0.5$ ), we found that the threshold corresponding to the best sensitivity-specificity pair is $44.4 \mathrm{pg} / \mathrm{mL}$. Our results showed that the potential of developing obesity is 16 times higher when the threshold serum ghrelin is less than $44.4 \mathrm{pg} / \mathrm{mL}$ with a sensitivity of $80 \%$ and a specificity of $80 \%$ (Table 3 ).

Table 3. Determination of risk corresponding to the threshold of ghrelin from the receiver operating characteristic (ROC) curve

\begin{tabular}{|c|c|c|}
\hline & Obese women & Group control \\
\hline \multirow[t]{2}{*}{$<44.4 \mathrm{pg} / \mathrm{mL}$} & 32 & 4 \\
\hline & $\mathrm{Se}=80 \%$ & $20 \%$ \\
\hline \multirow{2}{*}{$\geq 44.4 \mathrm{pg} / \mathrm{ml}$} & 8 & 16 \\
\hline & $20 \%$ & $\mathrm{Sp}=80 \%$ \\
\hline Total & 40 & 20 \\
\hline
\end{tabular}

Se = Sensitivity, $\mathrm{Sp}=$ specificity; $\chi^{2}=20 ; P<0.001 ; \mathrm{OR}=16,95 \%$ confidence interval 4.2 to 61 


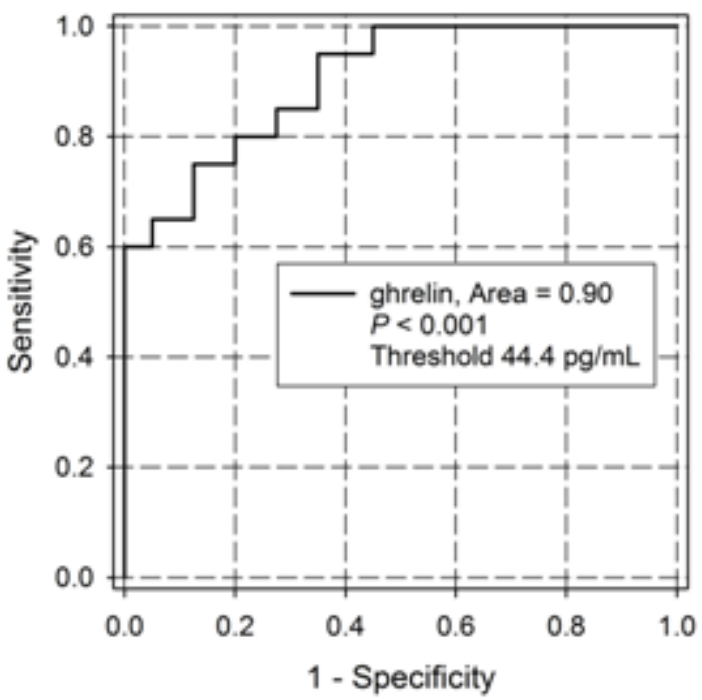

Figure 4. Receiver operating characteristic curve for ghrelin and obesity

After identifying the correlations between ghrelin, BMI, leptin, glucose, HDL-cholesterol and LDLcholesterol, we performed a multivariate linear regression analysis using backward method. We introduced ghrelin as the dependent variable and BMI, blood glucose, HDL-cholesterol, LDL-cholesterol and leptin as explanatory variables. Our results showed that HDL-cholesterol, LDL-cholesterol, and blood glucose are significantly associated with ghrelin in obese women.

\section{Discussion}

We showed that circulating levels of HDLcholesterol and ghrelin were significantly lower in the obese group. The plasma level of leptin was proportional to fat mass quantities and ghrelin, and seem to be modulated by the state of energy balance. To remedy obesity, there should be an increased production of leptin and a decreased production of ghrelin, thus reducing food (caloric) intake and increasing energy expenditure.

\section{Correlation between plasma leptin, plasma ghrelin, and BMI}

We found a significant positive correlation between plasma levels of leptin and BMI in the two groups (obese: $r=0.61, P<0.001$; controls: $r=0.95$, $P<0.001)$. These results are consistent with those of Sanlier [11] and other authors [13-20]. The correlation was consistent with that found by Ibrahim et al. [21] and Ezam et al. [22].

Our results confirm the existence of a significant negative correlation between plasma levels of ghrelin and BMI for both groups studied (obese: $r=-0.41$, $P=0.01$; controls: $r=-0.61, P=0.005)$. These results are consistent with those of Tschop et al. [23], Monti et al. [17], Koc et al. [19], Hamed et al. [24], Suematsu et al. [25], Purnell et al. [26], Al-Hakeim and Ali [27], Saad et al. [28], and Stepien et al. [29] (in a group of hypertensive subjects with class II and III obesity). Indeed, ghrelin is synthesized in inverse proportion to energy balance: the highest values are found in malnourished patients, lower values in lean subjects, and the lowest values were observed in obese patients. This apparently challenging adaptive response to inhibiting appetite depends on the deregulation of the energy balance [30]. However, Eizadi et al. [31] showed that ghrelin levels are independent of BMI. This is consistent with our multivariate analysis in obese women, which showed that there is no direct correlation between these two variables, suggesting that the correlation established in the univariate analysis is dependent on other variables. Indeed, changes in BMI are accompanied by proportional changes in leptin and insulin. In the obese, there is a downregulation of ghrelin, which correlates inversely with leptin and insulin [22, 27]. The interference of these variables explains the lack of direct correlation between ghrelin and BMI in the multivariate analysis.

\section{Correlation between plasma leptin, plasma ghrelin and plasma glucose}

A significant positive correlation was found between leptin and glucose levels in the two groups (obese: $r=0.41, P=0.008$; controls: $r=0.53$, $P=0.017)$. These findings are consistent with the 
findings of Hamed et al. [24] in obese patients with diabetes. However, our results are in discordance with several studies. Indeed, Martins et al. [32] did not identify any significant correlation between leptin and glucose. They concluded that hyperinsulinemia and insulin resistance strongly influence leptin. They even found that leptin is the best predictive parameter of insulin resistance in both sexes. Similarly, Taghdir et al. [33] found no significant correlation between leptin and fasting plasma glucose in the groups studied, and found a significant positive correlation between leptin and insulin, and leptin and insulin resistance in the control group. In the same context, Baban et al. [34] concluded that there was a significant positive correlation between circulating levels of fasting leptin and insulin. Silha et al. [13] have demonstrated a significant positive correlation between leptin and fasting insulin and a clear link between leptin and fasting glucose without having a significant correlation.

Our data also showed a significant negative correlation between circulating levels of ghrelin and glucose in the obese group. These results are consistent with those found by Purnell et al. [26] and Kellokoski et al. [35]. By contrast, Eizadi et al. [36] and Zwirska-Korczala et al. [37] failed to find a correlation between these two variables. This discrepancy may be related to differences between the various populations studied. Despite the lack of concordance between the results of our univariate analysis and data from the literature, this direct correlation persisted after the completion of the multivariate analysis.

\section{Correlation between plasma leptin, plasma ghrelin, and cholesterol}

Circulating levels of leptin showed a significant positive correlation with LDL-cholesterol in both groups (obese: $r=0.48, P=0.011$; controls: $r=0.61$, $P=0.005$ ). Haluzik et al. [38] has established no correlation between leptin and LDL-cholesterol both in a hyperlipidemic group and control group. We found that the level of leptin correlates negatively with HDL-cholesterol. This correlation was significant for both groups studied (obese: $r=-0.48, P=0.001$; control group: $r=-0.50, P=0.024)$. These results are consistent with those found by Ibrahim et al. [21], Ezam et al. [22], Saad et al. [28], and Haluzik et al. [38].

Plasma levels of LDL-cholesterol are negatively correlated with those of ghrelin, but significant only for the obese group $(r=-0.42, P=0.011)$. These results were consistent with findings by Ezam et al. [22] and Saad et al. [28] in their control group (nonobese/nondiabetic, but not in nondiabetic/obese and diabetic/obese groups). The correlation between ghrelin levels and LDL-cholesterol could be explained by presence of hyperglycemia in the obese group. This suggests the existence of insulin resistance. However, the size of the control group $(n=20)$ may also explain the lack of significant correlation. In addition, multivariate analysis has verified the existence of a direct correlation between ghrelin and LDLcholesterol. Indeed, a direct link between these two variables is not well established, but Kellokoski et al. [35] explained it by the potential ability of ghrelin to increase the binding of oxidized LDL in macrophages and monocytes, thereby inducing a decrease in its free level. Moreover, we found a significant positive correlation between plasma levels of ghrelin and HDLcholesterol and for the two groups (obese: $r=0.64$, $P=0.001$; controls: $r=0.48, P=0.032$ ). These results are consistent with those found by Ezam et al. [22], Purnell et al. [26], Al-Hakeim and Ali [27], Saad et al. [28], and Nogueira et al. [39]. Multivariate analysis found that there is a direct correlation between ghrelin and HDL-cholesterol levels. The mechanism of these two influencing variables is not known, but Beaumont et al. [40] have demonstrated that ghrelin may bind to HDL-cholesterol particles, which serve as its circulatory carrier, and which would explain this correlation.

\section{Correlation between plasma leptin and plasma ghrelin}

We found that leptin was significantly and negatively correlated with ghrelin in both groups (obese: $r=-0.5, P=0.001$; controls: $r=-0.58$, $P=0.007)$. These findings are consistent with those of Tschöp et al. [23] and Monti et al. [17] in a population of 233 people (107 men and 126 women) ranging in age from 23 to 75 years, and Ko et al. [19]. Krzyzanowska-Swiniarska et al. [41] found a negative correlation between ghrelin and leptin in an obese noninsulin-resistant group. The insulin-resistant obese group shows no correlation as such. The presence of this correlation in the noninsulin-resistant obese group and its absence in the obese insulinresistant group is consistent with results found in multivariate analysis, which did not result in a correlation between leptin and ghrelin. Leptin is 
synthesized in proportion to the state of adiposity and it acts by inhibiting neuropeptide Y/agouti related neuropeptide and proopiomelanocortin neurons by stimulating the cocaine- and amphetamine-regulated transcript. Ghrelin levels decreased in obesity suggest that there is a downregulation of ghrelin as an adaptive response to obesity [23]. With the presence of such a correlation, leptin appears to be involved in the negative regulation of ghrelin. A limitation of our study is that we did not measure insulin. However, insulin seems to be involved in the pathophysiology of obesity and correlates with the parameters we studied.

\section{Conclusions}

Further studies are needed to explore the characteristics of the Tunisian population and to better understand the variables in the development and dynamics of obesity in Tunisian women.

We declare that there is no conflict of interest in our work.

\section{References}

1. Xi B, Chandak G R, Shen Y, Wang Q, Zhou D. Association between common polymorphism near the MC4R gene and obesity risk: a systematic review and meta-analysis. PLoS One. 2012; 7:1-7.

2. Sartipy P, Loskutoff D J. Monocyte chemoattractant protein 1 in obesity and insulin resistance. PNAS. 2003; 100:7265-70.

3. World Health Organization. [online]. 2013 [cited 2013 October 2]; 311:4 pages. Available from http://www. who.int/mediacentre/factsheets/fs311/en/

4. Kanter R, Caballero B. Global gender disparities in obesity: a review. Adv Nutr. 2012; 13:491-8.

5. Beltaifa L, Ben Alaya N, Gaigi S, Delpeuch F. The causal model of obesity in Tunisia. In: Sahar AY (ed), G Le Bihan (coord). The causal approach applied to food and nutrition surveillance in Tunisia. Montpellier: CIHEAM, 2002. P. 71-93 (Mediterranean options: Series B. Studies and Research No. 41).

6. National Institute of Nutrition and Food Technology (Institut National de Nutrition et de Technologie Alimentaire, INNTA). Evaluation de l'etat nutritionnel de la population tunisienne. Enquete Nationale 1996/ 1997. Rapport National, Ministere de la Santè Publique. Tunis [online] 2000 [cited 2013 October 2]; 312 p. [in French] Available from http://www. institutdenutrition.rns.tn/index.php?option $=\mathrm{Com}_{-}$ content\&task=section\&id=63\&Itemid $=113$
7. Sahu A. Leptin signaling in the hypothalamus: emphasis on energy homeostasis and leptin resistance. Front Neuro. 2003; 24:225-53.

8. Sato T, Nakamura Y, Shiimura Y, Ohgusu H, Kangawa $\mathrm{K}$, Kojima M. Structure, regulation and function of ghrelin. J Biochem. 2012; 151:119-28.

9. Valassi E, Sacacchi M, Cavagnini F. Neuroendocrine control of food intake. Nutr Metab Cardio Dis. 2008; 18:158-68.

10. Arora S, Anubhuti. Role of neuropeptides in appetite regulation and obesity: a review. Neuropeptides. 2006; 40:375-401.

11. Sanlier N. The effect of body composition on blood lipids, leptin, bone mineral density, and nutrition in female. Saudi Med J. 2008; 29:1636-42.

12. Geray N. Endocrine controls of eating: CCK, leptin and ghrelin. Physiol Behav. 2004; 81:719-33.

13. Silha JV, Krsek M, Skrha JV, Sucharda P, Nyomba BL, Murphy LJ. Plasma resistin, adiponectin and leptin levels in lean and obese subjects: correlations with insulin resistance. Eur J Endocrinol. 2003; 149:331-5.

14. Benazzouz B, Hajaji Y, Hafidi A, Oudrhiri M, Boutayeb S, Guerre-Millo M, et al. Relation entre la concentration plasmatique de leptine et les paramètres anthropomètriques dans une population marocaine de sujets obèses. Biologie et Santè. 2004; 4:67-76. [in French]

15. Das P, Bhattacharjee D, Bandyopadhyay SK, Bhattacharya G, Singh R. Association of obesity and leptin with insulin resistance in type 2 diabetes mellitus in Indian population. Indian J Physiol Pharmacol. 2013; 57:45-50.

16. Vaidya R, Shringi M, Vaidya A, Godse C, Nanavati P, Shah $\mathrm{S}$, et al. Serum leptin concentrations in hyperinsulinemia in the sets of obese, lipodystrophic and/or non-obese patients. J Assoc Physicians India. 1999; 47:584-8.

17. Monti V, Carlson JJ, Hunt SC, Adams TD. Relationship of ghrelin and leptin hormones and the body mass index, the waist circumference in a random sample if adults. J Am Diet Assoc. 2006; 106:822-8.

18. Al Maskari MY, Alnaqdy AA. Correlation between serum leptin levels, body mass index and obesity in Omanis. Sultan Qabus Univ Med J. 2006; 6:27-31.

19. Koc F, Tokac M, Kocabas V, Kaya C, Buyukbas S, Erdem S, et al. Ghrelin, resistin and leptin levels in patients with metabolic syndrome. Eur J Gen Med. 2011; 8:92-7.

20. Gorpeliglo C, Erdal E, Ardicoglu Y, Adam B, Sarifakioglu E. Serum leptin, atherogenic lipids and glucose levels 
in patients with skin tags. Indian J Dermatol. 2009; 54: 20-2.

21. Ibrahim AE, El Yassin HD, Al Janab HKS. The association between leptin with lipid profile and tropinin in acute myocardial infraction patients. Iraqi Postgrad Med J. 2011; 10:332-8.

22. Ezam AK, Samy W, Hassanian MA. Ghrelin, leptin, insulin and insulin resistance in newly diagnosed type 2 diabetic patients. Benha M J. 2007; 24:189-90.

23. Tschop M, Viswanath D, Weyer C, Tataranni PA, Ravussin E, Meimann ML. Circulating ghrelin levels are decreased in human obesity. Diabetes. 2001; 50:707-9.

24. Hamed EA, Zakary MM, Ahmed NS, Gamal RM. Circulating leptin and insulin in obese patients with and without type 2 diabetes mellitus: relation to ghrelin and oxidative stress. Diabetes Res Clin Pract. 2011; 94:434-41.

25. Suematsu M, Katsuki A, Sumida Y, Gabazza EC, Murashima S, Matsumoto K, et al. Decreased circulating levels of active ghrelin are associated with increased oxidative stress in obese subjects. a clinical study. Eur J Endocrinol. 2005; 153:403-7.

26. Purnell JQ, Weigle DS, Breen P, Cummings DE. Ghrelin levels correlate with insulin levels, insulin resistance, and high-density lipoprotein cholesterol, but not with gender, menopausal status, or cortisol levels in humans. J Clin Endocrinol Metab. 2003; 88: 5747-52.

27. Al-Hakeim HK, Ali M. Low ghrelin level is associated with poor control and bad prognosis parameters in obese diabetic patients. J Diabetol. 2012; 1:5-14.

28. Saad MA, Kabalan YM, Al-Quobaili FA. Serum ghrelin levels in Syrian obese patients with diabetes mellitus type II. Afr J Biochem Res. 2013; 7:1-7.

29. Stęppien M, WlazeI RN, Paradowski M, Banach M, Rysz M, Misztal M, et al. Serum concentrations of adiponectin, leptin, resistin, ghrelin and insulin and their association with obesity indices in obese normo- and hypertensive patients - pilot study. Arch Med Sci. 2012; 8:431-6.

30. Zac-Vrghese S, Tan T, Bloom SR. Hormonal interactions between gut and brain. Discov Med. 2010; 10:543-2.

31. Eizadi M, Khorshidi D, Doali H, Valiollah S. Comparison of basal ghrelin concentration in type 2 diabetic with healthy men and association with BMI.
IPCBEE. 2011; 5:404-6.

32. Martins MC, Faleiro LL, Fonseca A. Relationship between leptin and body mass and metabolic syndrome in an adult population. Original Article. Rev Port Cardiol. 2012; 31:711-9.

33. Taghdir M, Djazayeri A, Djalali M, Eshraghian M, Rajab A, Yegane H.S, et al. Relationship of serum leptin concentration with insulin, glucose, HBA1c levels and insulin resistance in overweight postmenopausal diabetic women. ARYA Atheroscl J. 2010; 5:175-80.

34. Baban RS, Kasar KAK, Al Karawi IN. Fasting glucose to leptin ratio as a new diagnostic marker in patients with diabetes mellitus. Oman Med J. 2010; 25:269-74.

35. Kellokoski E, Kunnari A, Jokela M, Makela S, Kesaniemi YA, Horrko S. Ghrelin and obestatin modulate early atherogenic processes on cells: enhancement of monocyte adhesion and oxidized low-density lipoprotein binding. Metab Clin Exp. 2009; 58:1572-80.

36. Eizadi M, Dooaly H, Seyedhoseini MA, Khorshidi D. Associating serum ghrelin with some indicative markers of type 2 diabetic in healthy obese men. Euro J Exp Bio. 2011; 1:202-5.

37. Zwirska-Korczala K, Konturek SJ, Sodowski M, Wylezol M, Kuka D, Sowa P, et al. Basal and postprandial plasma levels of PYY, ghrelin, cholecystokinin, gastrin and insulin in women with moderate and morbid obesity and metabolic syndrome. J Physiol Pharmacol. 2007; 58:13-35.

38. Haluzik M, Fiedler J, Nedvidkova J, Ceska R. Serum leptin concentrations in patients with hyperlipidemia: Relationship to serum lipids and lipoproteins. Physiol Res. 1999; 48:363-8.

39. Nogueira JP, Maraninchi M, Beliard S, Lorec AM, Berthet B, Dubois N, et al. Unacylated ghrelin is associated with the isolated low HDL-cholesterol obese phenotype independently of insulin resistance and CRP level. Nutr Metab. 2012; 9:17.

40. Beaumont NJ, Skinner VO, Tan TM. Ramesh BS, Byrne DJ, Mac Coll GS, et al. Ghrelin can bind to a species of high density lipoprotein associated with paraoxonase. J Biol Chem. 2003; 278:8877-80.

41. Krzyzanowska-Swiniarska B, Kempa A, Miazgowski T, Pilarska K. Serum acylated ghrelin, adiponectin and leptin in normal weight and obese premenopausal women. Horm Metab Res. 2007; 39:835-9. 